\title{
EMBARAZO ABDOMINAL AVANZADO: DIAGNÓSTICO Y MANEJO. REPORTE DE UN CASO Y REVISIÓN DE LA LITERATURA
}

\section{Advanced abdominal pregnancy: Diagnosis and} management. Case report and review of the literature

\author{
Julie Angélica Mora-Enríquez, $M D^{1}$; Mortimer Arreaza-Graterol, $\mathrm{MD}^{2}$; \\ Hugo Hernán Nossa-Moreno, $\mathrm{MD}^{3}$; Jorge Augusto Rodríguez-Ortiz, $\mathrm{MD}^{4}$ \\ Recibido: marzo 28/14 - Aceptado: septiembre 12/14
}

\section{RESUMEN}

Objetivo: reportar el caso de un embarazo abdominal avanzado y realizar una revisión de la literatura sobre diagnóstico, manejo y pronóstico.

Materiales y métodos: se presenta el caso de un embarazo abdominal avanzado, controlado en la Unidad de Medicina Materno Fetal del Hospital Simón Bolívar, hospital público de tercer nivel de complejidad ubicado en Bogotá (Colombia). Se realizó una búsqueda de literatura médica en bases de datos Medline vía PubMed, y Lilacs, con termi-

1 Médica General, Universidad Tecnológica de Pereira. Residente de Tercer año de Ginecología y Obstetricia, Universidad de La Sabana, Hospital Universitario de La Samaritana, Bogotá (Colombia). jamedoc@hotmail.com

2 Especialista en Ginecología y Obstetricia, Universidad del Bosque. Especialista en Medicina Materno Fetal, Fundación Universitaria de Ciencias de la Salud. Profesor Ad Honorem Posgrado Ginecología y Obstetricia, Universidad de La Sabana, Unidad de Medicina Materno Fetal, Hospital Simón Bolívar, Bogotá (Colombia).

3 Especialista en Ginecología y Obstetricia, Universidad del Rosario. Ginecólogo oncólogo, Instituto Nacional de Cancerología. Profesor Titular, Posgrado Ginecología y Obstetricia, Universidad del Bosque, Unidad de Oncología Ginecológica, Hospital Simón Bolívar, Bogotá (Colombia).

4 Especialista en Ginecología y Obstetricia, Universidad del Bosque. Epidemiólogo Clínico, Universidad del Bosque. Profesor Titular, Posgrado Ginecología y Obstetricia, Universidad del Bosque. Profesor Ad Honorem Posgrado Ginecología y Obstetricia, Universidad de La Sabana, Unidad de Medicina Materno Fetal, Hospital Simón Bolívar, Bogotá (Colombia). nología MeSH "abdominal pregnancy" y "ectopic pregnancy”. La búsqueda se limitó a los idiomas inglés y español, entre los años 1940 a 2013.

Resultados: se encontró un total de 22 artículos relacionados directamente con el tema, de los cuales se incluyen 14 en la revisión: 1 artículo de revisión, 2 artículos de serie de casos, 9 reportes de caso y 2 cartas al editor.

Conclusión: el embarazo abdominal cercano al término es una situación obstétrica infrecuente que puede acarrear problemas severos para el binomio materno-fetal. El escenario ideal es poder realizar un diagnóstico temprano y su inmediata finalización.

Palabras clave: embarazo abdominal, embarazo ectópico.

\section{ABSTRACT}

Objective: To report a case of advanced abdominal pregnancy and conduct a review of the literature on the diagnosis, management and prognosis of this condition.

Materials and methods: A case of advanced abdominal pregnancy seen at the Mother and Child Care Unit of Hospital Simón Bolívar, a Level III public hospital in Bogotá, Colombia. A search was 
conducted in the medical literature through the Medline via PubMed and Lilacs databases, using the MeSH terms "abdominal pregnancy" and "ectopic pregnancy". The search was limited to the English and Spanish languages, and to the time period between 1940 and 2013.

Results: A total of 22 articles directly related to the topic were found, 14 of which were included in the review: 1 review article, 2 case series report, 9 case reports and 2 letters to the editor.

Conclusion: An abdominal pregnancy close to term is an infrequent obstetric situation that may result in severe problems for the mother and the foetus. The ideal scenario is an early diagnosis and immediate termination.

Key words: Abdominal pregnancy, ectopic pregnancy.

\section{INTRODUCCIÓN}

El embarazo abdominal se conoce como la implantación del óvulo fertilizado en la cavidad peritoneal, excluyendo trompa, ovario o ligamentos (1). Adicionalmente, se define como embarazo abdominal avanzado cuando se evidencia la presencia de feto vivo en la cavidad después de las veinte semanas de gestación (2). Ocasionalmente, se clasifica como primario o secundario. Para considerar que el embarazo es primario (o verdadero) debe cumplir con los criterios de Studdiford descritos en 1942 (3): las dos trompas y los ovarios deben estar normales, sin evidencia de trauma reciente o remoto; no debe existir evidencia de fístulas útero-peritoneales, y el embarazo debe estar confinado exclusivamente en la superficie peritoneal sin relación con la fimbria tubárica que implique la posibilidad de una implantación secundaria después de una nidación en la trompa. El lugar de implantación más común, en el $55 \%$ de los casos, es el fondo de saco posterior, seguido del mesosalpinx, el epiplón y el peritoneo de las paredes abdominal o pélvica (3).

El embarazo abdominal es una complicación obstétrica con resultados adversos maternos y fetales. Se estima que la frecuencia de presentación de embarazo abdominal es de 10,9 por cada 100.000 nacidos vivos, y una tasa de 9,2 por cada 1000 embarazos ectópicos (4), con una tasa de mortalidad materna que oscila entre el $0,5 \mathrm{y}$ el $18 \%$, y una tasa de mortalidad perinatal del 40 al $95 \%$ (5). El riesgo de muerte materna por embarazo abdominal es 7,7 veces mayor con respecto al embarazo tubárico y 89,9 veces mayor que en un embarazo normal (6).

En la literatura mundial, desde 1946 hasta la fecha, se han descrito 164 casos asociados a embarazo abdominal, con diferentes abordajes, desenlaces, métodos de seguimiento, manejo posquirúrgico de la placenta in situ y complicaciones maternas y fetales. En Colombia solo se registran dos casos de embarazo abdominal reportados, uno de ellos es un embarazo a término, con feto vivo y posterior sobrevida del neonato (7), y otro caso de litopedio (8). La relevancia clínica asociada al embarazo abdominal no radica en la rareza de la presentación, sino que se trata de una complicación obstétrica de alto riesgo, por lo que su diagnóstico y manejo deben ser conocidos por los médicos generales y obstetras. Por este motivo, reportamos el presente caso diagnosticado y seguido por la Unidad de Medicina Materno Fetal del Hospital Simón Bolívar (Bogotá, Colombia), para recordar y revisar aspectos importantes en el manejo de esta complicación obstétrica.

\section{PRESENTACIÓN DEL CASO}

Paciente de 36 años de edad, G3P1E2V1, remitida de zona rural del departamento de Boyacá (Colombia), por hallazgo incidental ecográfico de embarazo abdominal avanzado de 25 semanas. Ingresa al Hospital Simón Bolívar, institución de cuarto nivel de complejidad, ubicado en Bogotá (Colombia), que atiende pacientes de bajo nivel socioeconómico afiliados al régimen de seguridad pública, para continuar con el seguimiento de la gestación bajo la supervisión de la Unidad de Medicina Materno Fetal. En el examen obstétrico se encontró: aspecto externo similar al embarazo intrauterino, abdomen 
globoso, altura de $27 \mathrm{~cm}$, feto vivo con partes fetales fácilmente palpables, longitudinal, cefálico, dorso anterior, frecuencia cardiaca 140 latidos por minuto. Sin dolor a la palpación abdominal. En el tacto vaginal se encontró cérvix desplazado hacia porción anterior y fondo de saco posterior abombado ocupado por polo cefálico.

Se realizó ecografía de control en la institución, encontrando feto vivo, extrauterino, longitudinal, ocupando el fondo de saco posterior, con desplazamiento del útero hacia la porción anterior, con movimientos fetales presentes, frecuencia cardiaca 156 latidos, peso fetal estimado 1123 g y valoración de la anatomía fetal limitada por anhidramnios (figura 1). Se confirmó el diagnóstico por RMN. No se lograron identificar plenamente las estructuras vasculares que nutrían la placenta, la cual se encontró hacia el lado izquierdo del abdomen y con Doppler color se reveló flujo vascular periférico (figura 2). Se sugirió a la paciente la terminación del embarazo, pero está no aceptó, por lo que se continuó el seguimiento estricto con intervalo semanal de forma ambulatoria hasta alcanzar las 34 semanas, edad gestacional en la que se definió finalizar la gestación por laparotomía previo esquema de maduración pulmonar. La intervención quirúrgica se planeó en conjunto con ginecología oncológica, información del caso a cirugía general y

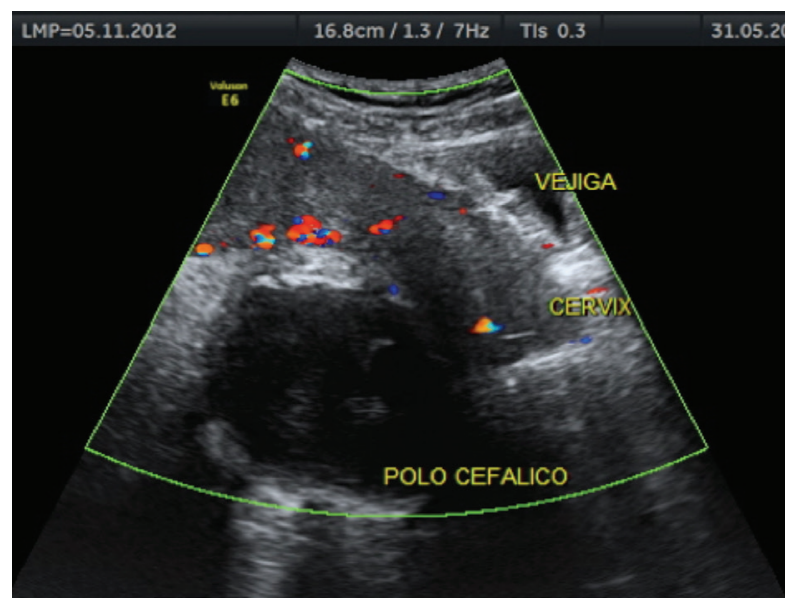

Figura 1. Relación vejiga, útero, polo cefálico neonatología, UCI neonatal, UCI adultos y reserva de hemoderivados, similar a lo establecido en protocolos de acretismo placentario. Bajo anestesia general se ingresa a la cavidad por incisión mediana supra e infraumbilical. Se identificaron fácilmente las membranas fetales (figura 3) incidiendo las mismas y extrayendo el feto sin dificultad, con polo cefálico ubicado en fondo de saco posterior con extracción de pelvis (figura 4). Se obtuvo un recién nacido vivo, femenino, peso de $2000 \mathrm{~g}$, Ballard para 34 semanas y puntaje Apgar de 3-46 al minuto 1,5 y 10 respectivamente. Se realizó revisión manual y se intentó establecer la ubicación de la placenta y su vascularización para definir la posibilidad de extracción. Sin embargo, se evidenció que la masa placentaria se encontraba firmemente adherida a la pared pélvica izquierda con grandes vasos a su alrededor, razón por la cual se definió intraoperatoriamente dejar la placenta in situ (figura 5). El procedimiento finalizó sin complicaciones. El recién nacido falleció cuatro horas después con diagnóstico de hipoplasia pulmonar severa y con varias alteraciones en su anatomía (dolicocefalia, aplanamiento facial, cuello corto, y pie equino varo bilateral, entre otras). No se pudo realizar estudio patológico por autopsia debido a la no autorización por parte de la madre y familiar. Para el manejo del tejido placentario se administraron tres ciclos

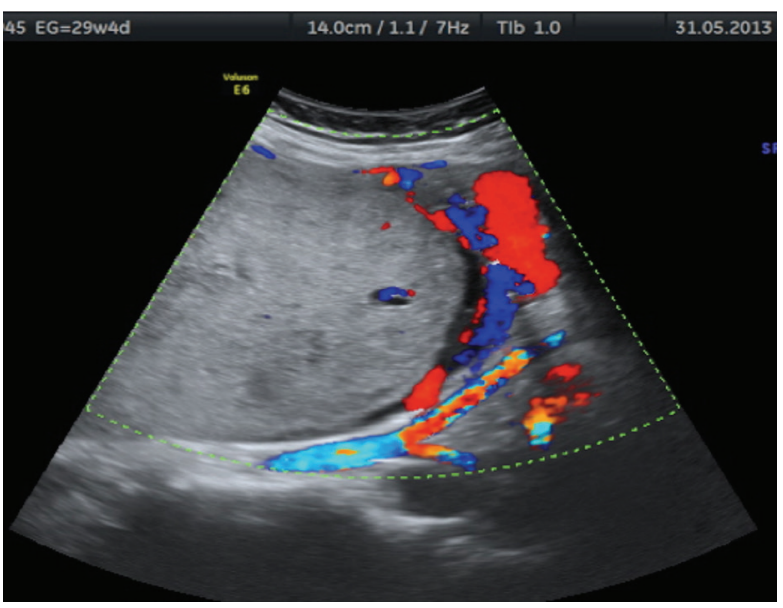

Figura 2. Placenta. Flujo lateral, imagen con Doppler color 


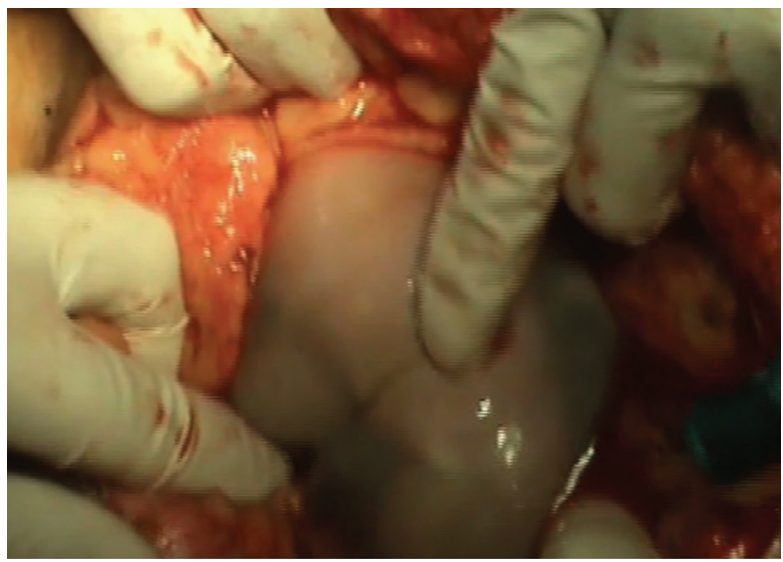

Figura 3. Identificación de bolsa amniótica

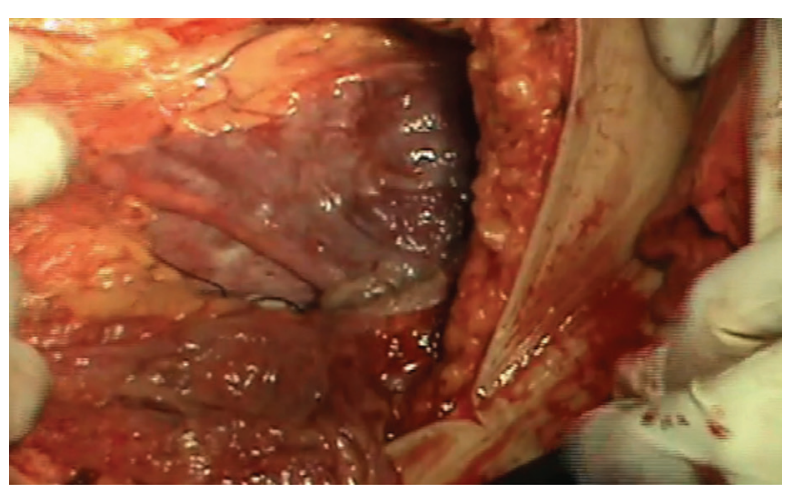

Figura 5. Placenta in situ

de metotrexato a dosis de $50 \mathrm{mg} / \mathrm{m}^{2}$ de superficie corporal con intervalos de tres semanas, logrando niveles negativos de BhCG a las seis semanas de la dosis inicial. Se complementó el seguimiento con ecografía cada tres semanas hasta completar dos meses de seguimiento, encontrándose el último diámetro registrado de la placenta de $15 \mathrm{~cm}$ y volumen aproximado de $640 \mathrm{cc}$. Actualmente, la paciente se encuentra estable, asintomática y sin complicaciones asociadas, en esquema de vigilancia semestral por el grupo de alto riesgo obstétrico.

Aspectos éticos. Se solicitó autorización por escrito a la paciente y al Hospital Simón Bolívar para publicar las fotografías. Se garantizó la confidencialidad de la información para proteger los derechos de la paciente.

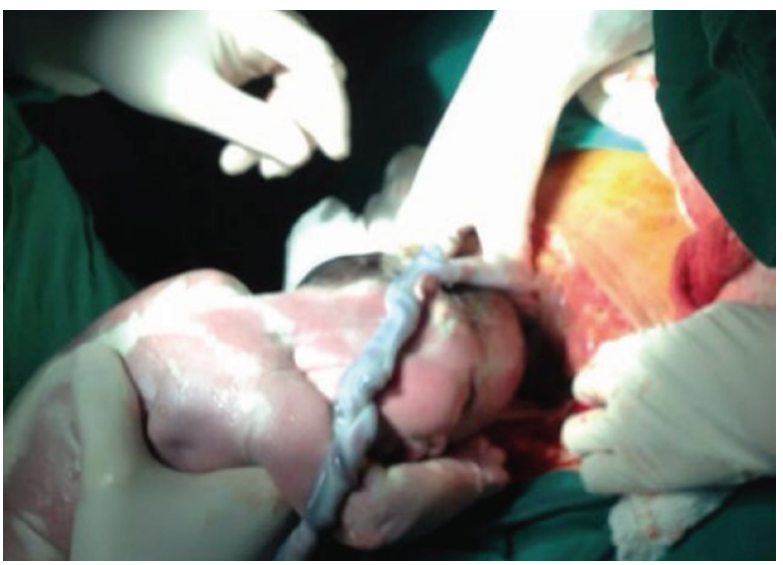

Figura 4. Finalización de la extracción en pelvis

\section{MATERIALES Y MÉTODOS}

Se realizó una búsqueda de la literatura médica en bases de datos Medline vía PubMed y Lilacs, con terminología MeSH "abdominal pregnancy" y "ectopic pregnancy", limitada a los idiomas inglés y español, entre los años 1940 a 2013.

\section{RESULTADOS}

Se encontraron 22 artículos, de estos se seleccionaron 14 para la revisión final del tema: una revisión del tema, 2 series de casos, 9 reportes de caso y 2 cartas al editor.

Diagnóstico. El curso clínico de la gestación abdominal varía desde el estado asintomático hasta el dolor abdominal difuso, síntoma más común $(3,9)$. Al examen físico se pueden encontrar presentaciones fetales anormales, anatomía fetal fácilmente palpable, ruidos cardiacos fetales más fuertes, fondo de saco de Douglas abombado, y cuello uterino cerrado y endurecido (3). La herramienta más importante para el diagnóstico del embarazo abdominal es la ecografía, con una sensibilidad de hasta el $99 \%$ en manos expertas (9). Los hallazgos ecográficos descritos en los casos de embarazo abdominal son: demostración del útero vacío adyacente a la vejiga, demostración de feto fuera del útero, reconocimiento del feto en aproximación estrecha con la pared abdominal materna, ausencia de miometrio alrededor del feto, 
posición fetal inusual, pobre definición de la placenta y oligohidramnios (9). Adicional a la valoración ecográfica, la resonancia magnética complementaria permite mejor visualización de las partes fetales, la placenta y el útero, y es útil a la hora de planear la intervención quirúrgica (10).

Los hallazgos del examen físico del caso presentado son similares a lo reportado en la literatura, basándose en la facilidad de la palpación de partes fetales y el abombamiento del fondo de saco de Douglas por ocupación del polo cefálico fetal. La herramienta de diagnóstico sin duda fue la ecografía, con claros hallazgos a la semana 25 , y pese a que la resonancia permite un mejor detalle del embarazo extrauterino (10), en este caso no aportó información adicional.

Tratamiento. Dada la rareza de los casos de embarazo abdominal, no existe un protocolo específico establecido por seguir $(1,3)$. El enfoque de manejo debe ir dirigido a finalizar la gestación una vez se realice el diagnóstico, lo cual en el presente caso se planteó pero no se realizó por la no aprobación materna, por lo cual se individualizó el caso, a pesar de las potenciales complicaciones materno-fetales y la poca posibilidad de sobrevida neonatal posterior reportada. El manejo por el que se optó en este caso, como en otros descritos (2, $6,11)$, fue dejar la placenta in situ y utilizar ciclos de metotrexate en búsqueda de la reabsorción del tejido placentario, debido a que la extracción de la misma no fue posible, reportándose respuestas tanto exitosas como fallidas con esta terapéutica (9). Si se deja la placenta in situ, se proponen dos tipos de manejo posterior: el expectante, con seguimiento ecográfico y de niveles de BhCG (8), o la administración de metotrexato en busca de acelerar la reabsorción del tejido como se refirió anteriormente (6). Se describen diferentes esquemas de metotrexato tales como: uso diario por cinco días (1), dosis semanal por dos dosis (4), dos dosis en los días 1 y 4 (12), y el utilizado en nuestro caso, referido por Jamal Rahaman, de 3 ciclos de metotrexato (50 mg/m² de superficie corporal) cada 3 semanas (13) logrando resultados semejantes de BhCG negativa luego de 3 dosis de administración. Por este motivo, el manejo médico dependerá de cada caso individual.

Pronóstico. Las principales complicaciones maternas reportadas son la hemorragia y la infección; sin embargo, el tiempo para desarrollarlas no se puede establecer con certeza (1). El principal determinante del pronóstico materno en el embarazo abdominal avanzado es la estrategia empleada para el manejo del tejido placentario. La tendencia general es dejar la placenta in situ en espera de la involución natural del tejido, ya que su extracción puede desencadenar un sangrado intraabdominal impredecible (8) porque no existe el control hemostático que ejerce la contracción miometrial sobre el lecho placentario, y los vasos que posee esta placenta se encuentran muy dilatados, mal conformados anatómicamente y muy penetrados a estructuras vecinas $(3,8)$. Sin embargo, si las condiciones son favorables, se puede optar por la extracción del tejido placentario (2) o, por otro lado, se reportan casos de histerectomía subtotal cuando la placenta se encuentra adherida al útero $(7,14)$. La descripción del manejo con respecto al tejido placentario se basó en los reportes de caso disponibles en la literatura. Está pendiente evaluar el resultado de las diferentes alternativas a largo plazo.

El pronóstico fetal infortunadamente es pobre y reservado en caso de encontrar una gestación avanzada; a lo largo de 60 años se han registrado muy pocos fetos vivos (15).

\section{CONCLUSIÓN}

El embarazo abdominal avanzado, además de constituir una situación obstétrica infrecuente, puede acarrear problemas severos al binomio maternofetal. El escenario ideal es poder realizar un diagnóstico temprano y su inmediata finalización. Hasta la fecha, el resultado del manejo materno es satisfactorio pero el pronóstico fetal es adverso. 


\section{REFERENCIAS}

1. Kevin C, Hnat M, Cunningham F. Advanced extrauterine pregnancy: diagnostic and therapeutic challenges. Am J Obstet Gynecol. 2008;198: 297. e1-297.e7.

2. Masukume G, Sengurayi E, Muchara A, Mucheni E, Ndebele W, Ngwenya S. Full-term abdominal extrauterine pregnancy complicated by post-operative ascites with successful outcome: a case report. Journal of Medical Case Reports. 2013;7:10.

3. Fylstra DL. Ectopic pregnancy not within the distal fallopian tube: etiology, diagnosis, and treatment. Am J Obstet Gynecol. 2012;206:289-99.

4. Shippey S, Bhoola S, Royek A, Long M. Diagnosis and Management of Hepatic Ectopic Pregnancy. Obstet Gynecol. 2007;109:544-46.

5. Atrash HK, Friede A, Hogue CJ. Abdominal pregnancy in the United States: frequency and maternal mortality. Obstet Gynecol. 1987;69:333-7.

6. Rahaman J, Berkowitz R, Mitty H, Gaddipati S, Brown B, Nezhat F. Minimally invasive management of an advanced abdominal pregnancy Obstet Gynecol. 2004;103:1064-8.

7. Díaz M. Embarazo abdominal a término: reporte de un caso. Rev Colomb Obstet Ginecol. 2002;53:93-5.

8. Iregui C, Borbón A, Cerquera F, García C. Litopedio. Diagnóstico por TC multicorte: reporte de un caso. Rev Colomb Radiol. 2009;20:2594-7.
9. Graham D, Johnson T, Sanders R. Sonographic findings in abdominal precnancy. J Ultrasound Med. 1982;1:7-14.

10. Koroglu M, Kayhan A, Soylu F, Erol B, SchimidTannawald C, Gurses C, et al. MR imaging of ectopic pregnancy with an emphasis on unusual implantation sites. Jpn J Radiol. 2013;31:75-80.

11. Zhang J, Li F, Sheng Q. Full-term abdominal pregnancy: a case report and review of the literature. Gynecol Obstet Invest. 2008;65:139-41.

12. Anderson P, Opfer E, Busch J, Magann E. An Early Abdominal Wall Ectopic Pregnancy Successfully Treated with Ultrasound Guied Intralesional Methotrexate: A Case Report. Obstet Gynecol Int. 2009;2009:247452.

13. Rahman MS, Al-Suleiman SA, Rahman J, Al-Sibai M. Advanced abdominal pregnancy-observations in 10 cases. Obstet Gynecol. 1982;59:366-72.

14. Norzilawati M, Shuhaila A, Siraj H, Mahdy Z, RashidZ. Advanced Abdominal Pregnancy Resulting from Late Uterine Rupture. Obstet Gynecol. 2008;111:502-4.

15. Roberts R, Dickinson J, Leung Y, Charles A. Advanced abdominal pregnancy: still an occurrence in modern medicine. Aust N Z J Obstet Gynaecol. 2005;45: 518-21. 